\title{
Effect of stellar rotation on oscillation frequencies
}

\author{
R.M. Ouazzani • M.J. Goupil • M.A. Dupret • D. Reese
}

Received: 17 September 2009 / Accepted: 28 January 2010 / Published online: 14 February 2010

(c) Springer Science+Business Media B.V. 2010

\begin{abstract}
We investigate whether the rotational splittings of $\beta$ Cephei stars can give some clue about the existence of a differential rotation in latitude, and if they are contaminated by the cubic order effects of rotation on oscillation frequencies. We also study some properties of splitting asymmetries and axisymmetric mode frequencies which provide seismic constrains on the distortion of the star. We find that only nonperturbative methods are able to reproduce those two seismic characteristics within $0.01 \%$ error bars for stars when they rotate faster than $3.3 \% \Omega_{k}$. If error bars of $1 \%$ are acceptable, the threshold of validity of perturbative methods is extended to $10 \% \Omega_{k}$.
\end{abstract}

Keywords Stellar rotation - Perturbative methods . Splitting $\cdot$ Differential rotation $\cdot$ Stellar oscillation $\cdot$ Beta Cephei

\section{Introduction}

$\beta$ Cephei stars are good candidates for seismic inferences, as these main sequence massive stars show low radial or-

R.M. Ouazzani $(\bowtie) \cdot$ M.J. Goupil · D. Reese

LESIA, Observatoire de Paris, 92195 Meudon, France

e-mail: rhita-maria.ouazzani@obspm.fr

R.M. Ouazzani

Université Pierre et Marie Curie, 75252 Paris, France

M.A. Dupret

Inst. d'Astrophysique et de Géophysique, Univ. de Liège,

4000 Liège, Belgium

D. Reese

Department of Applied Mathematics, University of Sheffield,

Sheffield S3 7RH, UK der modes which probe the deepest layers, can be identified, and for which rotational splittings are detected. As they are fast rotators, one must investigate what effects stellar rotation has on seismic diagnostic tools, and how it affects the information on stellar interior to which they give access.

For each oscillation frequency, $\omega_{0}$, from a non-rotating star, there corresponds $2 \ell+1$ frequencies, $\omega_{n, \ell, m}$ in rotating stars, where $n$ is the radial order, $\ell$ the degree and $m$ the azimuthal order. The basic splitting $S_{m}=\left(\omega_{n, \ell, m}-\omega_{n, \ell, 0}\right)$ is contaminated by the effect of asphericity, which introduces an antisymmetric component in the frequency as a function of $m$. We choose to remove this second-order contribution using $S_{m}^{g}=\left(\omega_{n, \ell, m}-\omega_{n, \ell,-m}\right) / 2 m$, the (generalised) splitting.

As a first approximation, this splitting is considered to be linear: $S_{m}=m \Omega_{0} \beta$, where $\Omega_{0}$ is the rotation rate, $\beta=C_{\ell}-1$, and $C_{\ell}$ the Ledoux constant (Dziembowski and Goode 1992, hereafter DG92). Departure from linearity arises when the rotation profile depends on the latitude. However, for fast rotators, such a departure could also be due to the cubic-order effects of rotation. What is the relative magnitude of these two effects? Can these two effects be easily disentangled?

Furthermore, in order to constrain stellar distortion, we can use axisymmetric mode frequencies and splitting asymmetries. Since in a perturbative approach, the effect of the centrifugal force arises mostly at the second order in the rotation rate, we neglect higher-order corrections. The second issue we address is whether for rapid rotation it is still reliable to use perturbative modeling, or if it is better to use nonperturbative methods - direct integration of a $2 \mathrm{D}$ eigenvalue system. 


\section{Basic theoretical framework}

The centrifugal force affects the structure of the star (causing distortion) and the resonant cavity of the modes is modified. The Coriolis force enters the equation of motion and affects the motion of the waves and frequencies of normal modes.

\subsection{Equilibrium configuration}

Following Chandrasekhar (1933), Chandrasekhar and Lebovitz (1962) the stationary equation of motion is solved by expanding the equilibrium quantities in terms of Legendre polynomials up to the second order. For the pressure for instance: $p(r, \theta)=p_{0}(r)+p_{22}(r) P_{2}(\cos (\theta))$, where $p_{0}$ includes the spherically symmetric deformation induced by rotation which is small (spherically symmetric part of centrifugal acceleration neglected here). The non-spherically symmetric part of the equilibrium quantities (such as $p_{22}$ ) are solved following Dziembowski and Goode (1992) (hereafter DG 92).

\subsection{Correction of frequencies up to the cubic order}

Following Soufi et al. (1998) (hereafter S98), the oscillation system is expanded up to the cubic order (see also Karami et al. 2005). Parts of the Coriolis and non-spherically symmetric distortion effects are included into the zeroth order of the oscillation equation. This way, we are able to solve the eigenvalue problem up to cubic order without having to solve the successive equations for the eigenfunctions at each order. Solving the eigensystem yields eigenfrequencies: $\omega=\omega_{0}+\omega_{c}$, where $\omega_{0}$ contains $\omega_{\Omega=0}$ and the Coriolis contribution to the first order, and $\omega_{c}$ represents the secondand third-order corrections to the eigenfrequencies due to the Coriolis and centrifugal forces. For more details, we refer to S98 and Karami (2008).

\section{Cubic-order frequency corrections}

Here we determine if a departure from linearity due to cubicorder effects can be significant and how it could affect the determination of the rotation rate. We compare it to what we consider as observational errors. As the observational error, we take here the resolution of a peak in the Fourier spectrum, for a CoRoT long (150 days) and short (20 days) runs.

\subsection{Corrections to the generalised splitting}

The splitting including frequency correction due to cubicorder effects and assuming a uniform rotation is given by

$S_{m}^{\text {cubic }}=\frac{\Omega_{0}}{\Omega_{k}} \beta+\frac{\Omega_{0}}{\sigma_{0}}\left(\frac{\Omega_{0}}{\Omega_{k}}\right)^{2} T_{m}$

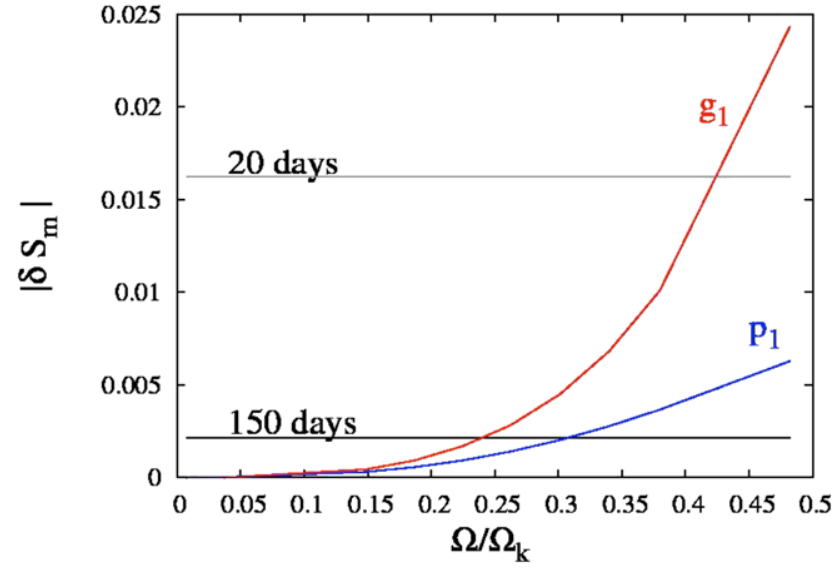

Fig. $1\left|\delta S_{m}\right|$ due to cubic-order effects, for a $g_{1}$ and a $p_{1}$ mode as a function of the rotational velocity. The two horizontal lines represent observational errors for CoRoT long and short runs respectively. All quantities are scaled by $\Omega_{k}$. Note that for $\mathrm{p}$ modes, this contribution is negative

where $\sigma_{0}=\omega_{0} / \Omega_{k}$ is the normalised frequency of the corresponding axisymmetric mode, and $\Omega_{k}=\sqrt{G M / R^{3}}$ is the break-up frequency. $T_{m}$ contains third-order contributions due to the Coriolis acceleration and distortion-which are dominant according to Karami et al. (2005) — as well as the coupling of the two. From now on, we define the departure from linear splitting as follows:

$\delta S_{m}^{\text {cubic }} \equiv S_{m}^{\text {cubic }}-\frac{\Omega_{0}}{\Omega_{k}} \beta=\frac{\Omega_{0}}{\sigma_{0}}\left(\frac{\Omega_{0}}{\Omega_{k}}\right)^{2} T_{m}$

We consider here as a representative model of $\beta$ Cephei star an $M_{\star}=8.5 M_{\odot}, R_{\star}=3.96 R_{\odot}$ model on the ZAMS. Cesam $2 \mathrm{k}$ has been used to build the internal structure and oscillations have been computed by the WarM (Warsaw Meudon oscillation) code.

Figure 1 shows that the cubic contribution to the splitting overtakes the observational error for a rotation rate above $23 \% \Omega_{k}$ (corresponding to a velocity of $v=R_{\star} \Omega=$ $150 \mathrm{~km} \mathrm{~s}^{-1}$ ) for a long run, and above $40 \% \Omega_{k}\left(260 \mathrm{~km} \mathrm{~s}^{-1}\right)$ for a short run. For $\beta$ Cephei stars, the mean rotation velocity is around $110 \mathrm{~km} \mathrm{~s}^{-1}$ (Stankov and Handler 2005), thus, for the majority of these stars, the cubic contribution does not significantly modify the splitting and one can use the linear splitting.

However, for faster rotators, we may wonder what impact neglecting third-order contributions has on the determination of a rotation rate from the generalised splitting.

\subsection{Impact on the determination of a rotation rate}

We consider that the splitting we measure from a real $\beta$ Ceph star is contaminated by third-order effects. Then one has 


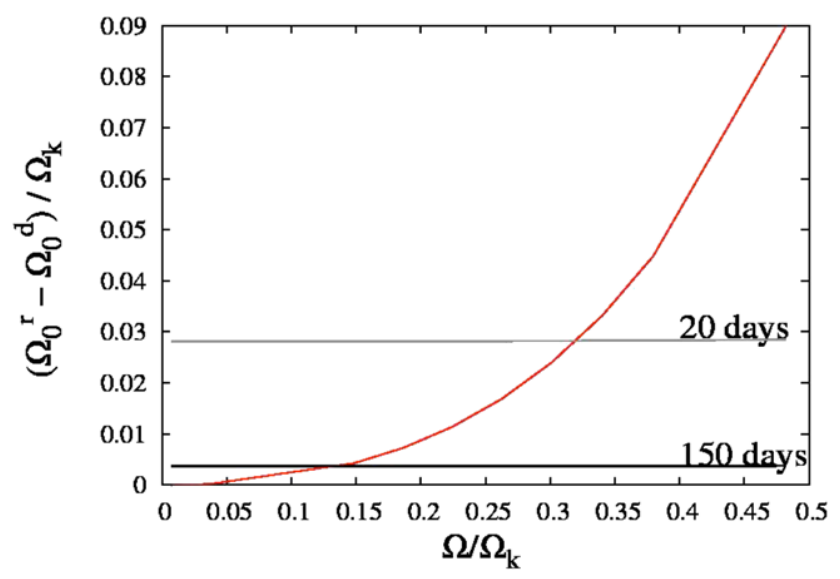

Fig. 2 Error on the determination of a rotation rate assuming linear splitting-i.e. neglecting third-order effects-as a function of the rotational velocity. The horizontal lines represent observational errors for CoRoT long and short runs. All quantities are scaled by $\Omega_{k}$

$S_{m}^{\text {obs }}=\frac{\Omega_{0}^{r}}{\Omega_{k}} \beta+\delta S_{m}^{\text {cubic }}$

where $\Omega_{0}^{r}$ is the assumed uniform rotation rate of the star, and $\delta S_{m}^{\text {cubic }}$ is given by (2). If the seismic analysis disregards cubic-order effects, then the rotation rate is extracted from the linear splitting:

$\Omega_{0}^{d}=\left(S_{m}^{\text {obs }} / \beta_{0}\right) \Omega_{k}$ where $\beta_{0}$ stands for the Ledoux constant calculated neglecting third-order effects. Then $\Omega_{0}^{d}$ departs from the true rotation rate $\Omega_{0}^{r}$ by

$\frac{\Omega_{0}^{r}-\Omega_{0}^{d}}{\Omega_{k}}=\frac{\Omega_{0}^{r}}{\Omega_{k}}\left(1-\frac{\beta}{\beta_{0}}\right)+\frac{1}{\beta_{0}} \delta S_{m}^{\text {cubic }}$

Figure 2 shows that for a long run, the resulting error on the determination of $\Omega_{0}$ is larger than the adopted observational error for rotation rates above $15 \% \Omega_{k}$. For our stellar model this corresponds to $100 \mathrm{~km} \mathrm{~s}^{-1}$. For a short run, an error of around $10 \%$ on determining the rotation rate becomes comparable to the observational error above $32 \% \Omega_{k}$ $\left(210 \mathrm{~km} \mathrm{~s}^{-1}\right)$.

\section{Origin of the departure from linear splitting}

Massive stars on the main sequence are fast rotators with a radiative envelope and shallow convection zones. An open issue is whether latitudinal differential rotation can exist in the envelope and if it can be measured. As mentioned before, cubic order and latitudinal differential rotation both induce a departure from the linear splitting. However, the effect of a latitudinal shear on a spectrum must not be mistaken with cubic-order effects. Cubic-order and latitudinal shear corrections are expected to show different behaviours when the rotation rate increases since the latitudinal differential rotation effect is proportional to the gradient of $\Omega$, whereas cubic-order effects are proportional to $\Omega^{3}$.
We then are led to investigate which of the two, cubicorder or the latitudinal gradient effect, is the most relevant to be taken into account, and in which rotation velocity range.

\subsection{Effect of latitudinal shear on the splitting}

We consider the same model as in Sect. 3.1. To compute the frequency in the case of a latitudinal differential rotation for the Sun, Dziembowski and Goode (1989) proposed a development of the rotation rate up the $4^{\text {th }}$ power in $\cos \theta: \Omega(\theta)=$ $\Omega_{0}+\Omega_{1} \cos ^{2} \theta+\Omega_{2} \cos ^{4} \theta$, where $\theta$ is the colatitude. They derived a rotational shear of $\Delta \Omega=\left(\Omega_{1}+\Omega_{2}\right) / \Omega_{0} \simeq 28 \%$ ( $\Delta \Omega=\Omega_{1} / \Omega_{0} \simeq 13 \%$ if we restrict the development to the $2^{\text {nd }}$ power of $\cos \theta$ ). Here, we assume a simple rotation law: $\Omega(\theta)=\Omega_{0}-\Omega_{1} \cos ^{2} \theta$ with $\Omega_{1}=\Delta \Omega$. Since $\Delta \Omega$ stands for $\Omega_{\text {equator }}-\Omega_{\text {pole }}$, it is positive when the equator rotates faster than the poles. For these main sequence massive stars, we consider values of $\Delta \Omega / \Omega_{0}$ ranging from $10 \%$ to $20 \%$. The effect of latitudinal differential rotation on frequencies have been derived by Gough and Thompson (1990) and DG92 (for more details we refer to Goupil 2010). One obtains

$S_{m}^{\text {latitude }}=\frac{\Omega_{0}}{\Omega_{k}} \beta_{0}\left(1-\frac{1}{5}\left(\frac{\Delta \Omega}{\Omega_{0}}\right)\right)$

where $\beta_{0}$ is $\beta$ obtained without taking into account thirdorder effects.

\subsection{Latitudinal dependence versus cubic-order effects}

The departure from linear splitting due to latitudinal differential rotation for $\ell=1$ is

$\delta S_{m}^{\text {lat }}=S_{m}^{\text {lat }}-\frac{\Omega_{0}}{\Omega_{k}} \beta_{0}=-\frac{1}{5} \frac{\Omega_{0}}{\Omega_{k}} \beta_{0}\left(\frac{\Delta \Omega}{\Omega_{0}}\right)$

Figure 3 compares the effects of latitudinal differential rotation and cubic-order effects. The first one is found to be dominant until $35 \% \Omega_{k}$ (for this stellar model: $250 \mathrm{~km} \mathrm{~s}^{-1}$ ). We recall that Stankov and Handler (2005) found a mean rotation velocity for $\beta$ Cephei stars of $110 \mathrm{~km} \mathrm{~s}^{-1}$. Then for the majority of $\beta$ Ceph stars, this effect should overtake cubic-order effects.

\subsection{How do we disentangle the two effects?}

The frequency spectrum of a rapidly rotating star is expected to present splittings which show departure from a linear splitting. How can we know that this is due to cubic-order effects or to latitudinal differential rotation?

Let $S_{m}^{\text {obs }}$ be the observed splittings for a fast rotator. Let the departure from linear splitting be due to cubic-order effects; then $S_{m}^{\text {obs }}=S_{m}^{\text {cubic }}\left(\Omega_{0}^{r}\right)$ according to (3). If one misinterprets $S_{m}^{\text {obs }}$ as due to a latitudinal shear, $S_{m}^{\text {obs }}$ is assumed to 


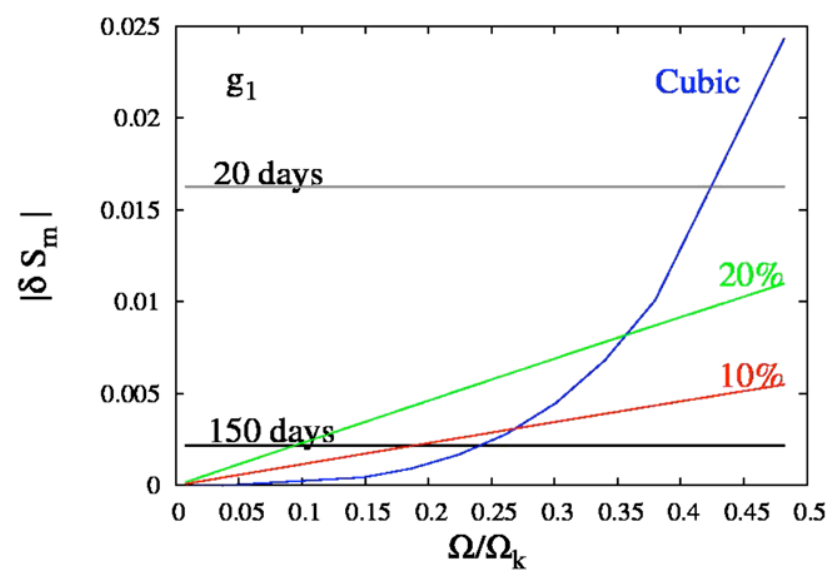

Fig. 3 Departure from a linear splitting versus the rotational velocity for a $g_{1}$ mode due to cubic-order effects (blue), to a $10 \%$ latitudinal shear (red) and to one of $20 \%$ (green). All quantities are scaled by $\Omega_{k}$. Note that the latitudinal contributions to the splitting are negative (6)

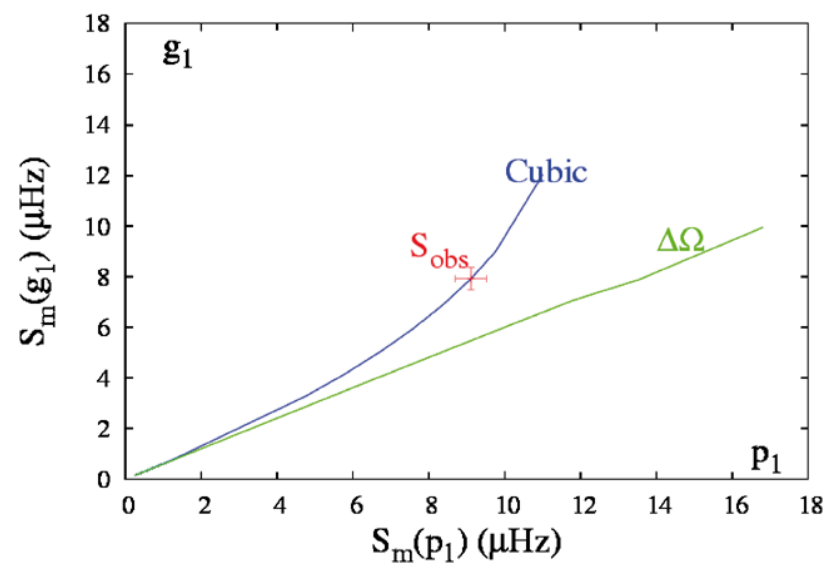

Fig. $4 S_{m}\left(g_{1}\right)$ versus $S_{m}\left(p_{1}\right)$ due to cubic-order effects in blue and to a latitudinal shear in green for an increasing rotation rate (from left to right)

obey (5). Note that for $g$ modes, $\beta_{0}$ is roughly equal to $1 / 2$, and for $\mathrm{p}$ modes $\beta_{0}$ approaches 1 .

Accordingly, the ratio between $S_{m}^{\text {lat }}\left(p_{1}\right)$ and $S_{m}^{\text {lat }}\left(g_{1}\right)$ does not depend on $\Delta \Omega$ and is approximately equal to 2 . In Fig. 4 $S_{m}\left(g_{1}\right)$ versus $S_{m}\left(p_{1}\right)$ is plotted for the two different assumptions (latitudinal shear or cubic-order effects) for different rotation rates $\left(\Omega \leq 35 \% \Omega_{k}\right.$ ). On the same plot we placed the two observed splittings of $g_{1}$ and $p_{1}$. Then as the observed point is not located on the straight line drawn by $S_{m}^{\text {lat }}\left(p_{1}\right)=2 S_{m}^{\text {lat }}\left(g_{1}\right)$, the departure is not due to a shear in latitude, but to cubic-order effects.

\section{Constraining the distortion with axisymmetric modes and splitting asymmetry}

The main effect on oscillation frequencies is due to the nonspherical part of the centrifugal distortion, and it is of second

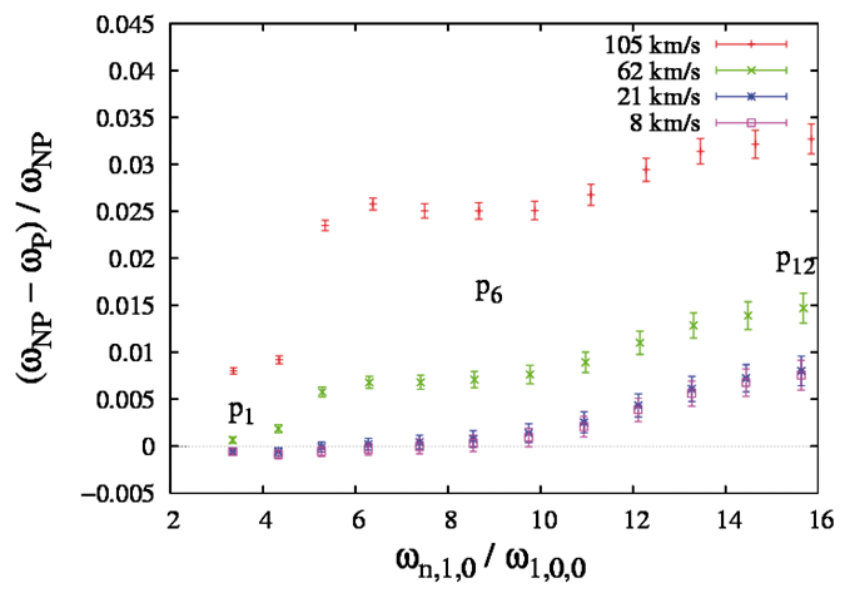

Fig. 5 Relative error induced on axisymmetric p mode frequencies by using a perturbative method compared with a non-perturbative one, with respect to the frequency which is scaled by $\omega_{1,1,0}$

order in terms of the rotation rate. To study this contribution, we neglect cubic-order effects and use a second-order perturbation formalism (DG 92). Neglecting near degeneracy, the oscillation frequency of a given mode can be written as in Goupil (2009):

$\omega_{n, \ell, m}=\omega_{0}+m \Omega \beta+\frac{\Omega^{2}}{\omega_{0}}\left(D_{0}+m^{2} D_{1}\right)$

where the second term on the right hand side is the asymmetry due to centrifugal distortion which dominates for high radial order modes. The issue we want to address here is if perturbative techniques are accurate enough to constrain efficiently distortion by seismic tools such as axisymmetric mode frequencies or splitting asymmetries. To do so we compare these two quantities using two different approaches: a second-order perturbative method, and the direct integration of a $2 \mathrm{D}$ eigenvalue system. The non-perturbative computations have been carried out following Reese et al. (2006). All the computations are made for an $M_{\star}=8.5 M_{\odot}$, $R_{\star}=3.96 R_{\odot}$, model of star, 20 Myrs old.

\subsection{Axisymmetric modes frequencies}

According to (7), axisymmetric $\mathrm{p}$ mode frequencies are given by $\omega_{n, \ell, 0}=\omega_{0}+\left(\Omega^{2} / \Omega_{k} \omega_{0}\right) D_{0}$. Knowing that $D_{0}$ behaves like $\left(\omega_{0} / \Omega_{k}\right)^{2}$, the second term of the right hand side behaves like $\left(\Omega / \Omega_{k}\right)^{2} \omega_{0} / \Omega_{k}$.

Figure 5 plots the differences between the two computations of axisymmetric $p$ mode frequencies for different rotation rates. Relative error bars are taken from Jerzykiewicz et al. (2005) and Suárez et al. (2009) for vEri, and multiplied by ten $(0.01 \%)$. It shows that as soon as we consider rotation rate higher than $\Omega_{T h}=3.3 \% \Omega_{k}, 20 \mathrm{~km} \mathrm{~s}^{-1}$, the discrepancies between the two approaches are no longer negligible compared to observational errors $(0.01 \%)$. In Reese 


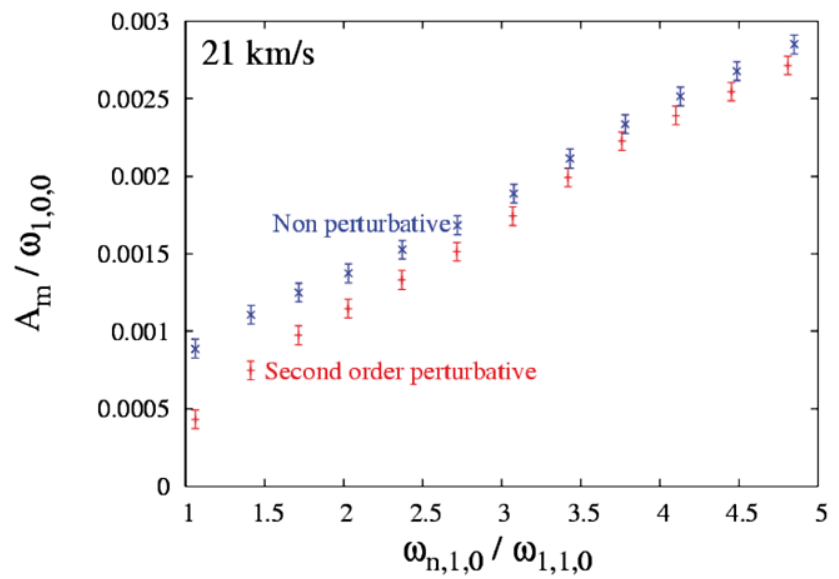

Fig. 6 Splitting asymmetry for $\mathrm{p}$ modes, scaled by $\omega_{1,1,0}$, as based on the perturbative method (in red), and on the non-perturbative methods (in green) for $21 \mathrm{~km} \mathrm{~s}^{-1}$

(2006), oscillation frequencies have been computed for an $N=3,1.9 M_{\odot}$ polytropic model, using a non-perturbative method, and compared to a fit of these frequencies using up to fifth-order polynomials in $\Omega$. He found that for secondand third-order polynomials, the differences between the frequencies computed with the two different methods are not negligible compared to the CoRoT short run error bar above $\Omega_{T h}=10 \% \Omega_{k}$, which here represents $71 \mathrm{~km} \mathrm{~s}^{-1}$. This validity threshold is lower when one calculates the polynomials coefficients using a perturbative method rather fitting these coefficients to the non-perturbative frequencies. This is no surprise, as fitting frequencies computed with a non-perturbative method captures several higher-order effects which are not included in a stricto sensu perturbative approach.

\subsection{Asymmetry}

One of the effects of rotation on oscillation frequencies is that the different components of a multiplet are no longer equally spaced. As done in DG 92, Suárez et al. (2009) and references therein, it is then convenient to define $A_{m}$ the splitting asymmetry as

$A_{m}=\omega_{0}-\frac{1}{2}\left(\omega_{m}+\omega_{-m}\right)=\frac{\Omega^{2}}{\Omega_{k} \omega_{0}} D_{1} \quad$ for $\ell=1$

Knowing that $D_{1}$ behaves like $\left(\omega_{0} / \Omega_{k}\right)^{2}, A_{m}$ behaves like $\left(\Omega / \Omega_{k}\right)^{2} \omega_{0} / \Omega_{k}$, which means that it linearly increases with $\omega_{n, \ell, 0}$. This is illustrated in Fig. 6 which presents the asymmetry computations as a function of mode frequency for the two different approaches. The radial order goes from $n=1$ to $n=12$. Once again, the error bars have been taken from
Jerzykiewicz et al. (2005), and multiplied by ten (0.01\%). In Fig. 6, we notice that for low rotation rates $\left(21 \mathrm{~km} \mathrm{~s}^{-1}\right)$, the behavior is quite similar but the discrepancies between the two approaches are still above the observational error bars of $0.01 \%$.

\section{Summary}

We have studied the cubic-order effects of rotation for an 8.5 $M_{\odot}$ ZAMS stellar model of star. We found that for the majority of $\beta$ Ceph stars, the cubic contribution does not have a significant impact on the splittings. Moreover, at high rotation velocities, it is in competition with the effect of latitudinal differential rotation. But if we dispose of two observed splittings, one for a $\mathrm{g}$ mode, and one for a $\mathrm{p}$ mode, then we can discriminate between the two hypotheses.

Furthermore, we studied axisymmetric mode frequencies and splitting asymmetries for an $8.5 M_{\odot}$ stellar model, 20 Myrs old. We find that if we accept a relative error of $1 \%$, then we can use the perturbative approach up to a rotation velocity of around $10 \% \Omega_{k}$ which here corresponds to $60 \mathrm{~km} \mathrm{~s}^{-1}$.

Acknowledgements This work was supported by the Centre National d'Etudes Spatiales (CNES).

\section{References}

Chandrasekhar, S.: Mon. Not. R. Astron. Soc. 93, 390 (1933)

Chandrasekhar, S., Lebovitz, N.R.: Astrophys. J. 136, 1082 (1962)

Dziembowski, W.A., Goode, P.R.: Astrophys. J. 540, 347 (1989)

Dziembowski, W.A., Goode, P.R.: Astrophys. J. 670, 394 (1992)

Gough, D.O., Thompson, M.J.: Mon. Not. R. Astron. Soc. 25, 242 (1990)

Goupil, M.J.: Lecture Notes in Physics, vol. 45, p. 765. Springer, Berlin (2009)

Goupil, M.J.: Lecture Notes in Physics. Springer, Berlin (2010, in press)

Jerzykiewicz, M., Handler, G., Shobbrook, R.R., Pigulski, A., Medupe, R., Mokgwetsi, T., Tlhagwane, P., Rodríguez, E.: Mon. Not. R. Astron. Soc. 619, 360 (2005)

Karami, K.: Chin. J. Astron. Astrophys. 8, 285 (2008)

Karami, K., Christensen-Dalsgaard, J., Pijpers, F.P., Goupil, M.J., Dziembowski, W.A.: arXiv:astro-ph/0502194 (2005)

Reese, D.: Ph.D. Thesis (2006)

Reese, D., Lignières, F., Rieutord, M.: Astron. Astrophys. 621, 455 (2006)

Soufi, F., Goupil, M.J., Dziembowski, W.A.: Astron. Astrophys. 911, 334 (1998)

Stankov, A., Handler, G.: VizieR Online Data Catalog 215, 80193 (2005)

Suárez, J.C., Moya, A., Amado, P.J., Martín-Ruiz, S., RodríguezLópez, C., Garrido, R.: Astrophys. J. 690, 1401 (2009) 